\title{
Molecular Dynamics and Tubulin Polymerization Kinetics Study on 1,14-Heterofused Taxanes: Evidences of Stabilization of the Tubulin Head-to-Tail Dimer-Dimer Interaction
}

\author{
Alessandro Contini, ${ }^{\text {a, } \$, *}$ Graziella Cappelletti, ${ }^{b, \sharp}$ Daniele Cartelli, ${ }^{\text {b }}$ Gabriele Fontana ${ }^{c}$ and Maria Luisa \\ ${ }_{5}$ Gelmi $^{\mathrm{a}}$
}

Received (in $X X X, X X X)$ Xth $X X X X X X X X X 20 X X$, Accepted Xth $X X X X X X X X X 20 X X$

DOI: $10.1039 / b 000000 x$

The effects on tubulin dynamics of paclitaxel, ortataxel and two recently developed taxol derivatives bearing a five-membered heterocyclic ring fused at the 1,14 position were analysed by means of 10 molecular dynamic simulations and MMPBSA approach. Tubulin polymerization kinetics and microtubule morphology assays were also conducted, providing support to computational results. In particular, it has been shown that the two recently developed 1,14-heterofused taxanes IDN5839 and IDN5798 are able to speed up the in vitro tubulin assembly by promoting the nucleation phase and to affect microtubule network in cell earlier than paclitaxel.

\section{${ }_{15}$ Introduction}

The microtubule-targeted drug paclitaxel (Taxol®, 1, Figure 1) is widely used in clinical practice for cancer therapy. ${ }^{1}$

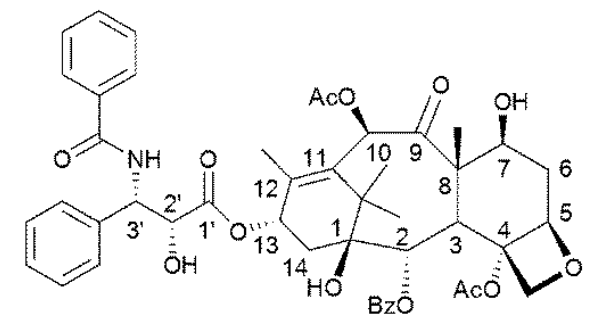

paclitaxel (1)

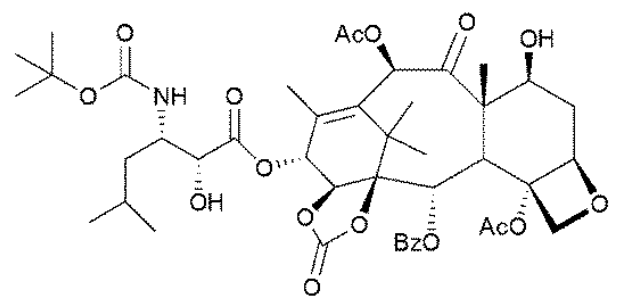

ortataxel (2)

Figure 1. Paclitaxel (1) and ortataxel (2)

20 Nevertheless, the research of analogues endowed by a more favourable pharmacological profile, in terms of selectivity and tolerability, is still necessary.

Structure-activity studies of several research groups leaded to the observation that changes to the "southern hemisphere", 25 comprising the $\mathrm{C} 14$ and $\mathrm{C} 1$ to $\mathrm{C} 5$ positions, exert a major effect on paclitaxel activity. ${ }^{2,3}$ As a demonstration, the potent anticancer taxoid ortataxel (2, Figure 1), was prepared starting from $14 \beta$ hydroxyl-10-deacetylbaccatin III 1,14-carbonate, a semi-synthetic intermediate obtained from the naturally abundant 10-deacetyl 30 baccatin III. $^{4}$ Ortataxel displays improved pharmacological properties, such as a remarkably higher oral bioavailability than paclitaxel and entered clinical trials where it showed a strong activity in Phase II studies on heavily pre-treated metastatic breast and non-small cell lung cancer. ${ }^{5}$ To further improve the 35 pharmacological properties of ortataxel, new C14 isosters were recently prepared by our research group. ${ }^{6}$

The development of highly active derivatives takes unquestionable advantages by the knowledge of bioactive conformations (Figure 2).

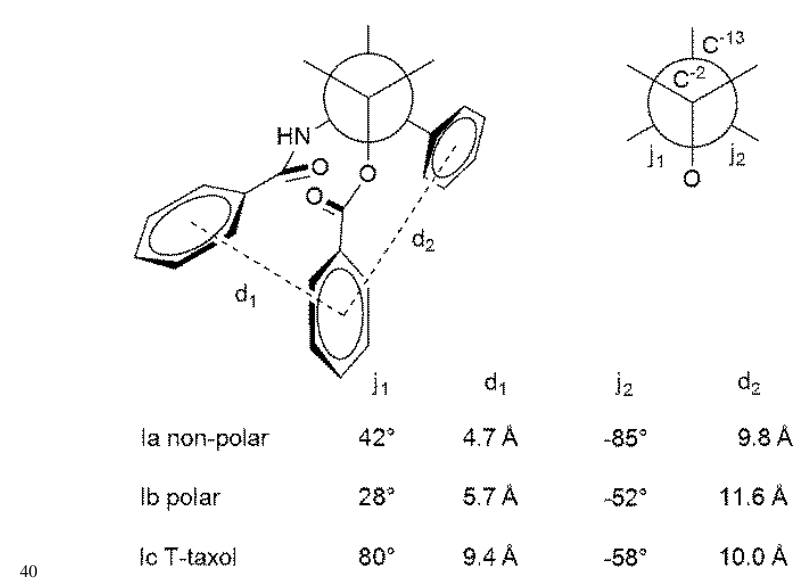

Figure 2. Proposed bioactive conformations for paclitaxel

The binding conformation of taxol and its derivatives has been the object of several investigations. A bioactive non-polar conformation Ia, showing the clustering of the $\mathrm{C} 2$ benzoate and 
the C3' benzamido group, was proposed by single conformation NMR studies in apolar solvents, ${ }^{7}$ while analogous NMR investigations conducted in polar solvents suggested the hydrophobically collapsed "polar" conformation $\mathrm{Ib} .{ }^{8}$ However, 5 most of the constrained taxol mimic designed upon both the nonpolar and the polar conformations resulted less potent than taxol itself. ${ }^{9}$ The T-taxol conformation Ic, supported by theoretical models derived from electron crystallographic density studies as well as by several experimental observations based on 10 constrained taxol analogues, ${ }^{10}$ was finally proposed as the bioactive conformation. ${ }^{11}$

Concerning taxol derivatives, the binding modes of IDN5390 (a seco-derivative of taxol) and epothilone A to type I and III isoforms of $\beta$-tubulin were investigated by means of restrained 15 molecular dynamic (MD) simulations, providing structural insights about the recognition mode of those antimitotics. ${ }^{12}$

MD simulations were also conducted on a microtubule fragment model (two parallel protofilaments formed by two $\alpha$ and one $\beta$ subunit) both in presence and absence of paclitaxel, ${ }^{13}$ 20 revealing through a root-mean-square-fluctuation analysis that paclitaxel induces changes in the dynamics of the loops surrounding the taxol binding site. The authors suggested that such changes, by affecting the protofilament flexibility, favour the interactions between adjacent protofilaments. Variations in 25 the dynamics at the $\beta-\alpha$ interface were also observed, but no details were given on the process thermodynamics and, consequently, on the eventual stabilization of the head-to-tail interaction between tubulin dimers in a single protofilament.

In the present work we investigated the binding mode and the 30 interaction energies for tubulin complexes of ortataxel (2) and the recently developed 1,14-heterofused taxanes IDN5839 (3) and IDN5798 (4) represented in Figure 3.

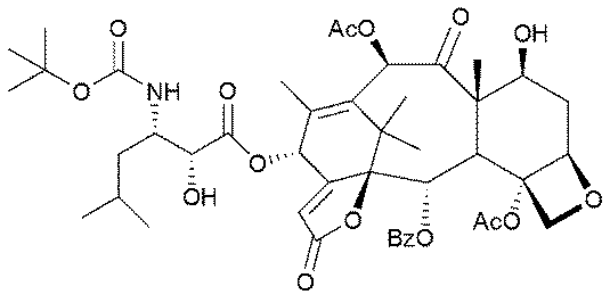

IDN5839(3)

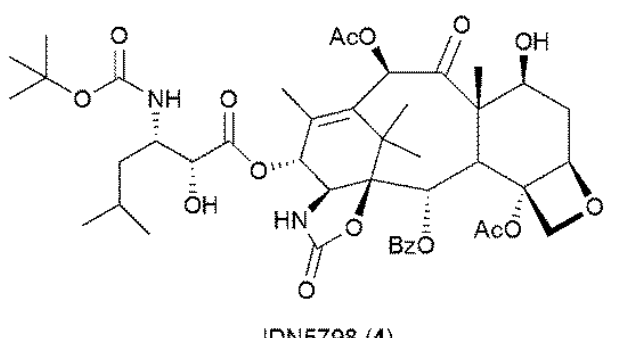

IDN5798(4)

Figure 3. 1,14-Heterofused taxanes analysed in the present study.

35 Paclitaxel was also included in the study for comparison and to test the reliability of our computational approach. Potential binding geometries for 1-4 were generated and then evaluated by MD simulations and molecular mechanics/Poisson Boltzmann surface area (MM-PBSA) calculations.
40 Moreover, the long range effects of taxanes binding on residues at the $1 \alpha-2 \beta$ interface were evaluated on $1 \beta, 1 \alpha-2 \beta, 2 \alpha$ tubulin tetramers by MD and MM-PBSA calculations, a method that proved to be successful in designing peptides interfering with the dimer-dimer interaction of tubulin, ${ }^{14}$ and per-residue free 45 energy decomposition analysis.

Finally, experiments on tubulin polymerization kinetics and on microtubule morphology were also conduced, and the comparison between theoretical and experimental findings revealed significant differences in the biochemical behaviour of the 50 investigated 1,14-heterofused taxanes.

\section{Results and Discussion}

\section{Computational Study.}

Binding mode and binding energy analysis. Although the structure of the $\alpha, \beta$-tubulin dimer complexed with 1 has been 55 determined by electron crystallography at $3.5 \AA{ }^{15}$ it lacks the necessary resolution to define the binding conformation of $\mathbf{1}$ itself. Despite this, the T-taxol bioactive conformation has been widely supported by binding studies with highly active constrained analogues ${ }^{10 \mathrm{a}, \mathrm{d}}$ and REDOR NMR experiments. ${ }^{11 \mathrm{c}}$ To 60 our knowledge, no information are available upon the bioactive conformation of 1,14-heterofused taxanes $\mathbf{2 - 4}$, and the assumption of an analogue binding mode with $\mathbf{1}$ might be dangerous due to the differences at the 1,14 positions and in the side-chain, which bears a t-butylcarbamate and a sec-butyl 65 instead of benzamide and phenyl groups.

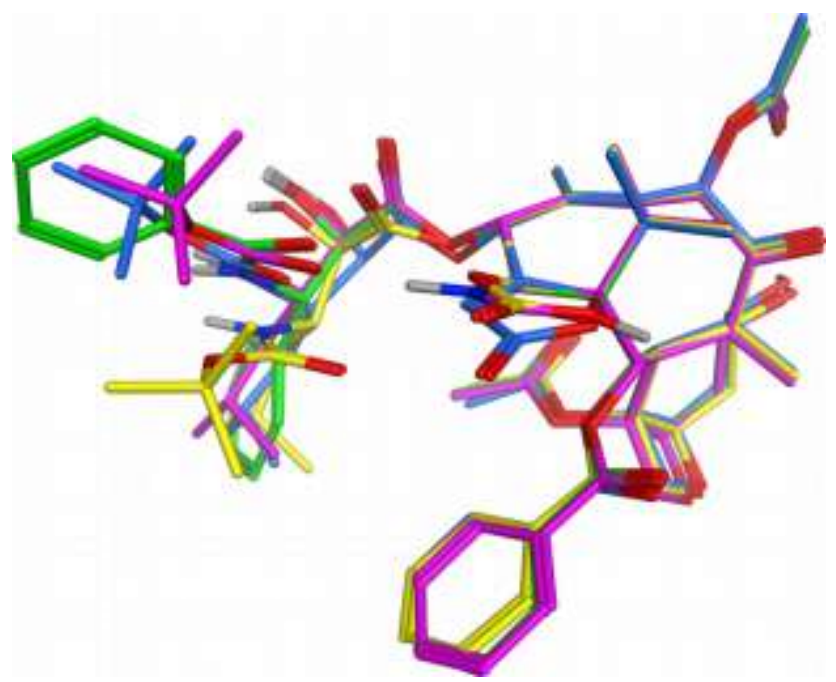

Figure 4. Superposed Lowest energy conformations obtained for the unbounded taxanes 1-4 by low-mode MD conformational search with the MMFF94x force field and Born solvation. 1 (green), 2 (yellow), 3 (cyan), 704 (magenta). Lowest energy conformations obtained for the unbounded taxanes by low-mode MD conformational search with the MMFF94x force field and Born solvation.

A conformational search of unbounded compounds 2-4 was then performed using the low mode MD algorithm and the 75 MMFF94x force field with Born solvation for water, implemented in the MOE software. ${ }^{16}$ Paclitaxel $\mathbf{1}$ was also included and the obtained conformation showed for this compound a geometry highly consistent with the REDOR-NMR supported T-taxol conformation. ${ }^{11 \mathrm{c}}$ Compounds 2-4 also provided 
comparable conformations, as shown by Figure 4 where the lowest energy conformations are superposed. Due to the structural differences in the side-chain and in the 1,14 region, the obtained conformation for $\mathbf{2 - 4}$ will be hereafter referred as 5 pseudo-T-taxol. It should be noted that, in the superposed geometries represented in Figure 4, the side-chain tertbutoxycarbonyl groups of compounds $\mathbf{3}$ and $\mathbf{4}$ and the side-chain benzoyl group of $\mathbf{1}$ are closely matched, while compound $\mathbf{2}$ shows an higher deviation (computed r.m.s.d. between the amidic 10 carbonyl groups of $\mathbf{2 , 3}$ and $\mathbf{4}$ compared to $\mathbf{1}$ are $0.14,0.11,0.12$ $\AA$, respectively). Although, by considering all the common heavy atoms in r.m.s.d. calculation between compounds $\mathbf{1}$ and $\mathbf{2 - 4}$, the closest match with $\mathbf{1}$ is observed for compound $\mathbf{2}$ (r.m.s.d. $=2.83$ A) followed by 4 and $\mathbf{3}$ (r.m.s.d. = 3.79 and $3.85 \AA$, respectively).

15 In order to evaluate the stability of the obtained conformations within the binding environment, complexes were realized by manually docking said conformations of 1-4 into the $\beta$ subunit of a tubulin dimer model derived from the $1 \mathrm{JFF}$ crystal structure. ${ }^{15}$ The complexes were processed by MD simulations in explicit 20 water and, after a careful equilibration, a 2 ns production run was performed, a length considered adequate in similar and recently published studies. $^{14}$

In order to verify the possibility of alternative binding conformations, different starting complexes were also generated 25 by automated docking, using two different protocols previously optimized in order to reproduce at best the experimentally determined binding conformation of $\mathbf{1}$ (see Experimental for details). For each compound, the top five poses of both protocols were used for generating complexes which were subjected to the 30 MD procedure described above. All trajectories were then analysed by MM-PBSA, a reliable method to discriminate different binding conformation by computing free energies of binding on a statistically relevant thermodynamic ensemble. ${ }^{17}$ In order to select a proper binding conformation, the MM-PBSA 35 derived complex absolute stabilities and ligand binding energies were both evaluated. Table 1 reports the binding energies obtained for the lowest energy pose (see Table S1, Supporting Information, for full results), together with geometrical parameters selected to provide a common term of comparison 40 between 1 and the 1,14-heterofused taxanes 2-4 (Figure 5).

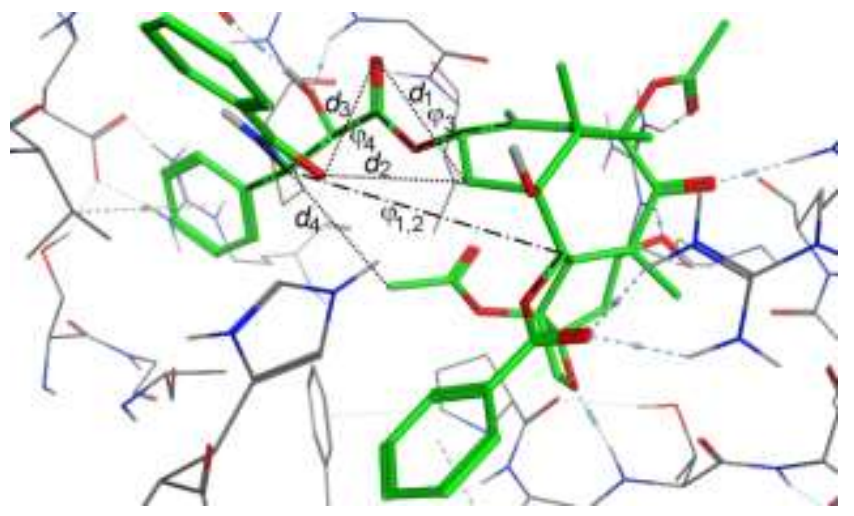

Figure 5. Distances $d_{1} \quad\left(\mathrm{Cl}^{\prime}=\mathrm{O} \cdots \mathrm{C} 14\right), \quad d_{2} \quad(\mathrm{C} 14 \cdots \mathrm{O}=\mathrm{CNH}), \quad d_{3}$ $\left(\mathrm{HNC}=\mathrm{O} \cdots \mathrm{O}=\mathrm{C}^{\prime}\right), d_{4}\left(\mathrm{C} 11-\mathrm{OCOCH} 3 \cdots \mathrm{C} 3{ }^{\prime}\right)$; torsion angles $\varphi_{1}$ and $\varphi_{2}$ (O-C2-C3'-N and O-C2-C3'-C, accordingly to reference 11a), and 45 dihedral angles $\varphi_{3}$ and $\varphi_{4}$ (C12-C13-O-C1' and O-C1'-C2'-C3', respectively, accordingly to reference $11 \mathrm{~b}$ ).
Table 1. Computed MM-PBSA Binding Energies for the Most Favored Pose and Average Geometrical Parameters for the Bounded Taxanes. ${ }^{a}$

\begin{tabular}{ccccc}
\hline & $\mathbf{1}$ & $\mathbf{2}$ & $\mathbf{3}$ & $\mathbf{4}$ \\
$\Delta E_{\mathrm{MM}-\mathrm{PBSA}}$ & $-39.7 \pm 7.0$ & $-36.0 \pm 5.4$ & $-36.6 \pm 5.4$ & $-29.2 \pm 4.1$ \\
$d_{1}$ & $3.9 \pm 0.2$ & $3.9 \pm 0.3$ & $3.8 \pm 0.2$ & $3.5 \pm 0.3$ \\
$d_{2}$ & $3.4 \pm 0.2$ & $4.5 \pm 0.4$ & $4.5 \pm 0.4$ & $4.1 \pm 0.4$ \\
$d_{3}$ & $3.8 \pm 0.3$ & $3.8 \pm 0.5$ & $3.8 \pm 0.4$ & $3.6 \pm 0.4$ \\
$d_{4}$ & $4.0 \pm 0.2$ & $4.2 \pm 0.3$ & $4.3 \pm 0.3$ & $3.9 \pm 0.2$ \\
$\varphi_{1}$ & $76.8 \pm 5.8$ & $85.7 \pm 9.7$ & $94.2 \pm 8.9$ & $61.7 \pm 12.2$ \\
$\varphi_{2}$ & $-56.9 \pm 7.4$ & $-70.5 \pm 13.5$ & $-59.7 \pm 9.2$ & $-85.8 \pm 14.9$ \\
$\varphi_{3}$ & $-117.5 \pm 18.2$ & $-112.3 \pm 23.8$ & $-114.0 \pm 16.9$ & $-139.7 \pm 23.1$ \\
$\varphi_{4}$ & $82.0 \pm 14.1$ & $94.7 \pm 19.0$ & $92.3 \pm 15.4$ & $95.5 \pm 14.8$
\end{tabular}

${ }^{\text {a }}$ Geometrical parameters averaged upon frames sampled in the final ns 50 trajectory of production run

As expected, the T-taxol conformation provided for $\mathbf{1}$ the best results in terms of both complex stability and ligand binding energy (Table S1, Paclitaxel(1) t-taxol). The same was observed for 3, where calculations starting from the pseudo-T-taxol 55 conformation provided the lowest energies in terms of stability and binding energy (Table S1, IDN5839(3) pseudo-T-taxol). Less clean results were obtained for compounds $\mathbf{2}$ and $\mathbf{4}$, where three low energy complexes were obtained for the former (IDN5109(2) 2_1, 2_2 and pseudo-T-taxol), and two for the latter (IDN5798(4) $605 \_2$ and pseudo-T-taxol). However, the pseudo-T-taxol conformation resulted in both cases the most favoured in terms of binding energies, confirming that $\mathbf{1}$ and the 1,14-heterofused taxanes 2-4 adopt similar binding modes.

The analysis of geometrical parameters (Figure 5 and Table 1) ${ }_{65}$ showed that $d_{1}, d_{3}$ and $d_{4}$ distances are quite conserved, while $d_{2}$ distance is evidently longer for 1,14-heterofused taxanes, probably due to the substitution at $\mathrm{C} 14$. Concerning selected torsions, describing the side-chain orientation relatively to the main scaffold, a slight widening of $\varphi_{1}$ and $\varphi_{4}$ dihedrals is 70 observed for compounds $\mathbf{2}$ and $\mathbf{3}$ with respect to $\mathbf{1}$, while only the former shows a widening of $\varphi_{2}$ of about $10 \mathrm{deg}$. A different behaviour is observed for compound $\mathbf{4}$, which presents a narrower $\varphi_{1}\left(61.7 \pm 12.2\right.$ deg.) and wider $\varphi_{2}$ and $\varphi_{3}(-85.8 \pm 14.9$ and $139.7 \pm 23.1$, respectively). This behaviour might be due to the 75 strong H-bond that can be formed between the side-chain tertbutyloxycarbonyl and the cyclic $\mathrm{NH}$ at $\mathrm{C} 14$, which characterizes compound 4.

The analysis of intermolecular H-bonds between $\beta$-tubulin and taxanes 1-4 (Table 2) shows interactions with Asp 26', Thr 274' 80 and Arg 282' for all of them. 1 also shows a rather persistent Hbond with Gln 280' and Gly 360', while the neighbouring Arg 359' establishes a quite stable and peculiar H-bond with 3 . Similarly to 1, 2 also establishes H-bonds with Gln 280' and Gly 360 ', even if their average occupancy is lower, while no H-bonds 85 are observed for compound 4 with Gln 280', Arg 359' or Gly 360 '. It should be noted that an H-bond, even if showing a low average occupancy (8.2\%), was also detected between the compound 3 carbonyl of the 1,14 ring and His 227'. 
Table 2. H-bond analysis. ${ }^{a}$ H-bonds with an average occupancy above $40 \%$ for at least one compound are reported

\begin{tabular}{|c|c|c|c|c|}
\hline \multirow[b]{2}{*}{ H-Bonds } & \multicolumn{4}{|c|}{ average occupancy $(\%)$} \\
\hline & 1 & 2 & 3 & 4 \\
\hline Asp26'-COO $\cdots \mathrm{HN}-\mathrm{C} 3{ }^{\prime}$ & 93.6 & 93.3 & 89.5 & 57.6 \\
\hline Asp26'-COO $\cdots \mathrm{HO}-\mathrm{C} 2{ }^{\prime}$ & 99.9 & 92.0 & 79.6 & n.d. \\
\hline Thr274'-NH $\cdots \mathrm{O}-\mathrm{C} 10$ & 96.1 & 88.5 & 65.1 & 26.0 \\
\hline $\mathrm{G} \ln 280^{\prime}-\mathrm{NH}_{2} \cdots \mathrm{O}=\mathrm{C} 6$ & 93.8 & 9.5 & n.d. & n.d. \\
\hline Arg282'- $\mathrm{NH}_{2} \cdots \mathrm{O}-\mathrm{C} 8$ & 17.8 & n.d. & 58.2 & 6.5 \\
\hline Arg282' $-\mathrm{NH}_{2} \cdots \mathrm{O}=\mathrm{C} 6$ & n.d. & n.d. & n.d. & 46.4 \\
\hline $\mathrm{rg} 282^{\prime}-\mathrm{NH}_{2} \cdots \mathrm{OC}\left(\mathrm{CH}_{3}\right) \mathrm{O}-\mathrm{C} 5$ & 59.6 & 25.0 & 17.2 & n.d. \\
\hline 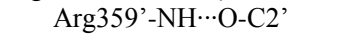 & n.d. & n.d. & 66.9 & n.d. \\
\hline 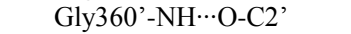 & 88.1 & 28.3 & n.d. & n.d. \\
\hline
\end{tabular}

${ }^{\text {a }}$ Parameters for H-bond analysis: donor-acceptor distance: 3.5 Å; donor$\mathrm{H}$-acceptor angle $120.0 \mathrm{deg}$. The analysis was performed on the last ns of 5 the production run trajectory.

MM-PBSA binding energies are comparable for all the investigated compounds, in line with previously reported results on cytotoxic activity $\left(\mathrm{IC}_{50}\right.$ on MCF7 tumor cell lines for $1,{ }^{18}$ and $2,3,4^{19}$ are $1.7 \pm 0.40,1.6 \pm 0.10,0.4 \pm 0.10$ and $1.0 \pm 0.04 \mathrm{nM}$, 10 respectively), even if, in discordance with experiments, compound 4 resulted the less favoured.

Analysis of the effects of taxanes at the tubulin $1 \alpha-2 \beta$ interface. Although we did not expect force field methods to discriminate ligands with such a narrow potency interval, the above 15 computational result might conceal a behaviour that goes farther than the simple differences in binding affinity. For this reason, we decided to test the effects of 1-4 on the interaction energy between two consecutive tubulin dimers, a closer model of a tubulin protofilament structure.

20 Table 3. MM-PBSA energy decomposition analysis for the proteinprotein interaction between tubulin $1 \alpha$ and $2 \beta$ subunits evaluated from the MD of $1 \beta, 1 \alpha-2 \beta, 2 \alpha$ tetramer; ${ }^{a}$ single contributions ( $\mathrm{kcal} / \mathrm{mol}$ ) for selected amino acids are reported relatively to unbounded tetramer. ${ }^{\mathrm{b}}$ Total MMPBSA binding energy $\left(\Delta E_{\text {binding }}, \mathrm{kcal} / \mathrm{mol}\right)$ for the $1 \alpha-2 \beta$ dimer-dimer 25 interaction is also reported..$^{\mathrm{c}}$

\begin{tabular}{cccccc} 
& \multicolumn{5}{c}{ per residue $\boldsymbol{\Delta} \boldsymbol{\Delta} \boldsymbol{E}_{\text {bound-unbound }}(\mathbf{k c a l} / \mathbf{m o l})$} \\
Chain & Residue & $\mathbf{1}$ & $\mathbf{2}$ & $\mathbf{3}$ & $\mathbf{4}$ \\
$1 \alpha$ & Arg2 & -0.4 & 0.0 & -3.7 & -5.6 \\
$1 \alpha$ & Lys163 & -0.3 & -0.2 & -0.6 & -4.2 \\
$1 \alpha$ & Glu254 & -2.1 & 0.7 & -0.2 & -2.0 \\
$1 \alpha$ & Asp345 & -1.1 & -1.7 & -0.3 & -2.0 \\
$1 \alpha$ & Pro348 & -2.3 & -1.1 & -1.1 & -1.0 \\
$1 \alpha$ & Thr349 & -0.8 & 2.0 & 0.1 & -1.6 \\
$2 \beta$ & Glu69', & 0.3 & 0.0 & -0.1 & -2.4 \\
$2 \beta$ & Arg390' & 3.6 & 2.4 & 3.5 & 3.6 \\
$2 \beta$ & Arg391, & 2.8 & 1.7 & 1.0 & 0.0 \\
$2 \beta$ & Phe394' & -1.4 & -2.4 & -0.9 & -3.2 \\
& & & & &
\end{tabular}
$\Delta E_{\text {binding }}$
$-39.1 \pm 5.6$
$-33.6 \pm 8.4$
$-52.1 \pm 6.5$
$-72.1 \pm 6.8$

a The analysis was performed on the last ns of the production run trajectory. ${ }^{\mathrm{b}}$ Single residue contribution are computed as the difference between taxane bounded and unbounded tubulin. Only contributions where $|\Delta \Delta E|>2 \mathrm{kcal} / \mathrm{mol}$ for at least one compound are reported. ${ }^{\mathrm{c}}$ The $30 \Delta E_{\text {binding }}$ computed for the unbounded tetramer is $-39.1 \pm 8.0 \mathrm{kcal} / \mathrm{mol}$.

MD simulations were performed on models of the $1 \beta, 1 \alpha-2 \beta, 2 \alpha$ tubulin tetramer, derived from the $3 \mathrm{~N} 2 \mathrm{G}$ tubulin tetramer crystal structure, ${ }^{20}$ unbounded or bounded with 1-4 in their most favoured binding poses (Figure $\mathrm{S} 1$ ).

35 The binding energy between the $1 \alpha-2 \beta$ subunits was then computed and decomposed into single residue contributions by MM-PBSA analysis of the last ns of production run trajectory. Computed relative energies with respect to unbounded tubulin, are reported in Table 3, while the full set of energies is provided 40 as Supporting Information (Table S2).

Unexpectedly, compound $\mathbf{4}$ provided the highest stabilization of the dimer-dimer interaction (MM-PBSA $\Delta E_{\text {binding }}=-72.1 \pm 6.8$ $\mathrm{kcal} / \mathrm{mol}$ ), followed by $\mathbf{3}$ which also provided a significant stabilization with respect to the unbounded tubulin $\left(\Delta E_{\text {binding }}=-\right.$ ${ }_{45} 52.1 \pm 6.5$ and $-39.1 \pm 8.0 \mathrm{kcal} / \mathrm{mol}$, respectively). On the other hand, neither compound 2 nor 1 significantly affected the $1 \alpha-2 \beta$ interaction energy. These data suggest that the principal microtubule stabilizing action of compounds $\mathbf{1}$ and $\mathbf{2}$ might occur by improving the parallel interaction between adjacent 50 protofilaments, as suggested by Mitra and co-workers, ${ }^{13}$ while an additional stabilization mechanism, exerted by improving the head-tail dimer-dimer affinity, could be expected for compounds 3 and 4.

\section{Experimental Study.}

55 In order to assess the interaction of compounds 1-4 with the tubulin/microtubule system, they were initially tested in an in vitro tubulin polymerization assay at fixed concentration of 10 $\mu \mathrm{M}$. As shown by the kinetics of assembly reported in Figure 6A, compounds 1-4 induce tubulin polymerization even in the 60 absence of GTP.

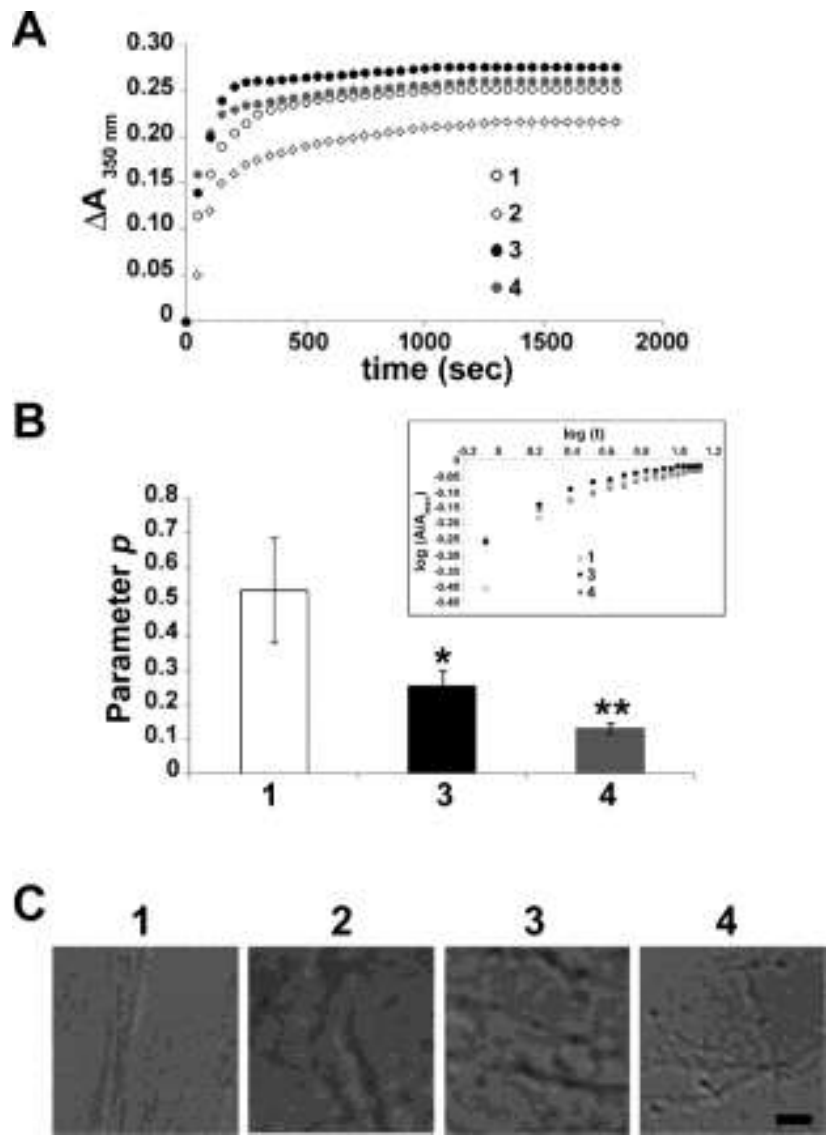

Figure 6. (A) Tubulin assembly was recorded as a function of time by measuring the increase in absorbance at $350 \mathrm{~nm}$. Tubulin $(13 \mu \mathrm{M})$ was polymerised in assembly buffer without GTP in the presence of 65 compounds 1-4 at $10 \mu \mathrm{M}$ concentrations. (B) Nucleation phase was analysed by extrapolating the parameter $\mathrm{p}$ from assembly kinetics, as shown in the inset, obtained in the presence of 1 or compounds 3,4 . (C) DIC microscopy images of microtubules assembled in the presence of compounds 1-4 (Bar, $2 \mu \mathrm{m})$. 


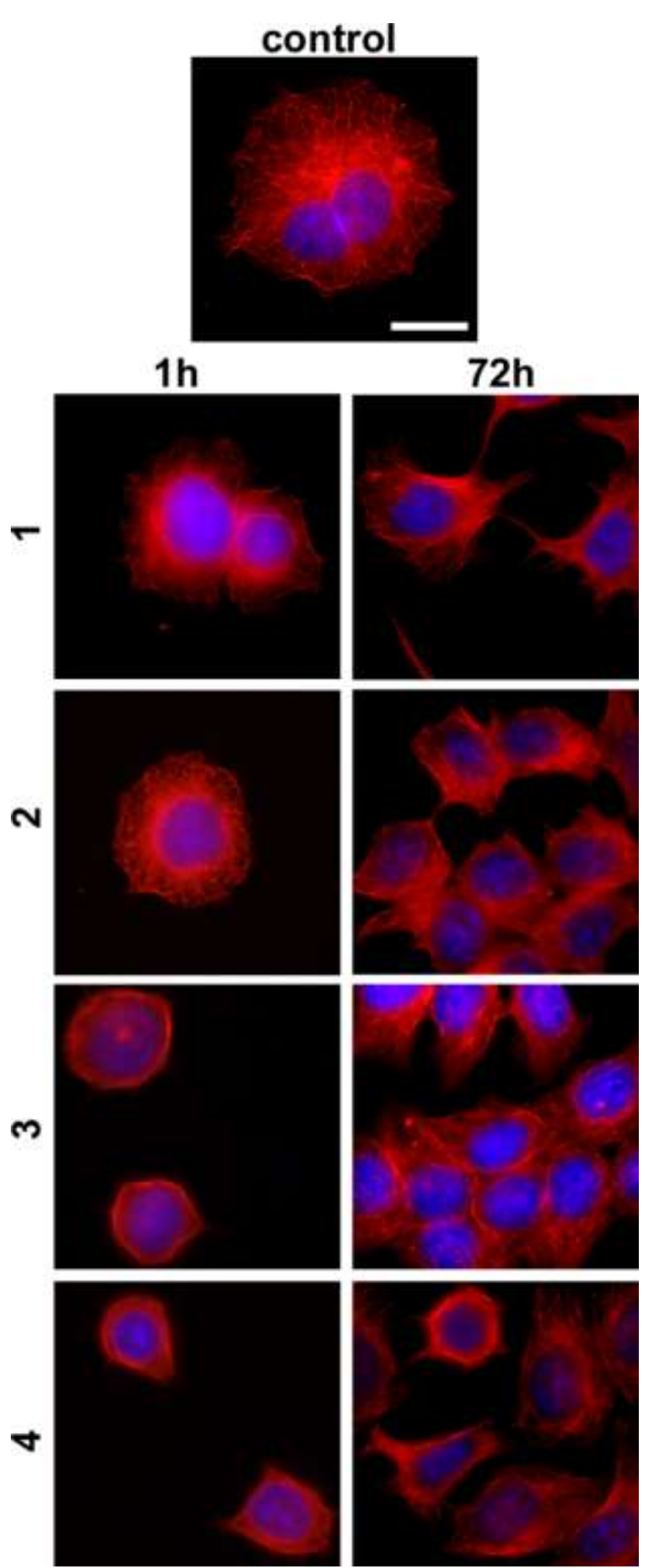

Figure 7. Microtubule organization in human MCF7 cells exposed for 1 and $72 \mathrm{~h}$ to compounds $\mathbf{1}(1.7 \mathrm{nM}), \mathbf{2}(1.6 \mathrm{nM}), \mathbf{3}(0.4 \mathrm{nM})$, and $\mathbf{4}(1 \mathrm{nM})$, as revealed by immunofluorescence localization of $\alpha$-tubulin (red). Nuclei 5 are stained by DAPI (blue). Scale bars, $20 \mu \mathrm{m}$

However, as the kinetics appear not to be perfectly superposed in the presence of the different compounds, we carried out further analyses to calculate a range of parameters characteristic of the assembly kinetics: nucleation, elongation, and steady state phase.
${ }_{10}$ From nucleation phase we extrapolated the parameter $p$ (see Experimental Section) and found that it was significantly reduced by the compounds $\mathbf{3}$ and $\mathbf{4}$ with respect to $\mathbf{1}$ (Figure 6B), suggesting these compounds as more effective than $\mathbf{1}$ in favouring microtubule nucleation.

15 Table 4. Kinetic parameters of tubulin assembly in the presence of compounds 1-4 $(10 \mu \mathrm{M})$.

\begin{tabular}{ccc}
\hline cmpd & $\mathbf{V}_{\mathbf{i}} \pm \mathbf{s e m}$ & $\mathbf{\Delta A} \pm \mathbf{s e m}$ \\
$\mathbf{1}$ & $0.074 \pm 0.029$ & $0.220 \pm 0.052$ \\
$\mathbf{2}$ & $0.081 \pm 0.026$ & $0.188 \pm 0.018$ \\
$\mathbf{3}$ & $0.159 \pm 0.040^{*}$ & $0.235 \pm 0.045$ \\
$\mathbf{4}$ & $0.158 \pm 0.027^{*}$ & $0.285 \pm 0.031$
\end{tabular}

* p $<0.05$ vs 1 according to ANOVA, Tukey post hoc.

Table 4 reports the maximal velocity of polymerization $(\mathrm{Vi})$ and the steady state extent of assembly $(\Delta \mathrm{A})$ extrapolated by the 20 assembly kinetics shown in Figure 6A. Compounds 3 and 4 induce a significant increase in $\mathrm{Vi}$ with respect to $\mathbf{1}$ showing the ability to improve the elongation phase as well as observed above for nucleation. Conversely, the steady state extent of assembly is comparable in the presence of $\mathbf{1}$ and all the tested compounds. ${ }_{25}$ Looking at microtubule morphology, we collected the assembled microtubules at the end of polymerization and analysed them by DIC microscopy (Figure 6C). Our results show that microtubules assembled in the presence of $\mathbf{1}$ and its derivatives show a similar morphology.

30 Finally, to investigate the effects of the compounds on microtubule organization in cells and, possibly, correlate these effects with their anti-proliferative activity, we have exposed MCF7 cells to $\mathbf{1 - 4}$ for 1 and 72 hours at concentrations corresponding to their $\mathrm{IC}_{50} \cdot{ }^{18,19}$ At the end of the treatment, cells 35 were fixed, microtubules stained with anti- $\alpha$-tubulin antibodies and observed by fluorescence microscopy (Figure 7).

In control cells, we observed a widespread network of long microtubules other than the typical accumulation of microtubules at one side of the nucleus called the microtubule organizing 40 centre (MTOC). Compound $\mathbf{1}$ induces the typical accumulation of microtubules in bundles at both 1 and $72 \mathrm{~h}$ of incubation times and a similar effect is evoked by $\mathbf{2}$. On the contrary, compounds 3 and 4 appear to induce a more dramatic reorganization of the microtubules at early time points: cells appear roundish after $1 \mathrm{~h}$ 45 of incubation with the above compounds and few bundles are detectable. To conclude, our in vitro results highlight that chemical modifications in derivatives $\mathbf{3}$ and $\mathbf{4}$ potentiate the wellknown taxol ability to influence tubulin assembly kinetics by improving nucleation and elongation. In addition, these 50 compounds evoked more severe effects on microtubule organization in MCF7 cells, in perfect agreement with our findings from the above reported computational study.

\section{Conclusions.}

The unbounded and bounded conformations of some biologically 55 active 1,14-heterofused taxanes were investigated by MD simulations and results were compared to the known postulated bioactive conformation of paclitaxel. We evidenced that compounds 2-4 present unbounded geometries comparable to the T-taxol model and these conformations, herein referred as 60 pseudo-T-Taxol, also resulted the most stable for the bounded 
taxanes. Interestingly, although the amido-derivative $\mathbf{4}$ showed some deviation from the pseudo-T-taxol conformation and also the lowest computed binding affinity, it provided the highest stabilization of the $\alpha 1-\beta 2$ interaction in a tubulin tetramer model, 5 followed by compound 3 .

The different behaviour of $\mathbf{3}$ and $\mathbf{4}$ with respect to ortataxel $\mathbf{2}$, also characterized by a 1,14-carbonyl bridge, might depends on the different physical-chemical features of the substituent at C14. Indeed, in compound $\mathbf{4}$ a strong hydrogen bond is formed 10 between the carbamic nitrogen and the carboxy group of BOC in the side-chain. In $\mathbf{2}$ such an attractive interaction is replaced by a repulsion between the same group and the oxygen of the carbonate. Compound $\mathbf{3}$ lies in the middle between $\mathbf{2}$ and $\mathbf{4}$, providing that the substitution of position 14 with methylidene 15 does not cause a repulsive or an attractive interaction.

Biological data consolidate the computational outcomes and highlight the significant impact of the 1,14-heterofused taxanes 3 and $\mathbf{4}$ on tubulin kinetics and organization. As $\mathbf{3}$ and $\mathbf{4}$ speed up in vitro tubulin assembly by promoting the nucleation phase, these 20 derivatives likely attest an improved ability to stabilize microtubules. Next, compounds $\mathbf{3}$ and $\mathbf{4}$ affect microtubule network in cell earlier than paclitaxel 1. Although we cannot exclude that the precocious microtubule collapse observed in cells exposed to compounds $\mathbf{3}$ and $\mathbf{4}$ could be amplified by 25 additional and not-microtubule dependant mechanisms, our data clearly show that chemical modifications in these derivatives augment paclitaxel performance in targeting tubulin organization in cell and, consequently, in inhibiting cell growth.

\section{Experimental Section}

${ }_{30}$ Computational Methods. Conformational searches for 1-4 were done using low-mode molecular dynamics with the MMFF94x force field and Born solvation, as implemented in the MOE software. ${ }^{16}$ The $\alpha, \beta$-tubulin structure was obtained from the 1JFF crystal structure ${ }^{15}$ by reconstructing missing residues through the 35 homology model module implemented in MOE. The model was then protonated with the $\mathrm{H}++$ server accordingly to a $\mathrm{pH}$ of 6.5 and a salinity of $0.15 \mathrm{M}^{21}$ and then relaxed by a force field minimization (Amber force field implemented in MOE) with backbone restraints (tether atom $=1000$ ). Docking were 40 performed with MOE and two protocols well reproduced the paclitaxel binding conformation as found in 1JFF. Protocol 1, using the Triangle Matcher algorithm with default settings and London $\mathrm{dG}$ scoring function, followed by force field refinement (MMFF94x) of the poses and rescoring (London $\mathrm{dG}$ ), provided 45 the best matching with the T-taxol conformation for the first pose (r.m.s.d. $=0.81 \AA$ ). Protocol 2, using the Alpha Triangle algorithm (800000 and 5000000 for minimum and maximum iteration, respectively, timeout $=6000$ seconds) and Affinity scoring function, followed by force field refinement and 50 rescoring (Affinity), provided an r.m.s.d. $=1.83 \AA$ for the first pose, but 0.81 and $1.00 \AA$ for the second and third, respectively. Both protocols were chosen, the first because it produced the best fitting for the top-ranked pose, the second because it was able to find the highest number of low r.m.s.d. geometries within the top${ }_{55}$ five poses. The top-five poses of compounds 1-4 were then used to generate $\alpha, \beta$-tubulin complexes for MD simulations. Analogue complexes were also prepared using the lowest energy conformation obtained by the previously described conformational search. All complexes were neutralized by adding ${ }_{60} 19 \mathrm{Na}+$ ions and solvated by a cubic TIP3P water box extending up to $10 \AA$ from the solute. Orientation and conformation independent RESP charges were derived for ligands 1-4 using the REDIII procedure on two conformations and two orientations, ${ }^{22}$ using the Gaussian03 software for ab-initio geometry 65 optimization and molecular electrostatic potential calculation. ${ }^{23}$ Parameters for GTP and GDP residues were downloaded from the Amber parameter database. ${ }^{24}$ MD simulations were performed with the pmemd module of the Amber11 program package using the ff03 and gaff force fields. ${ }^{25,26}$ All complexes were minimized, 70 heated up to the final temperature of $300 \mathrm{~K}$ by six consecutive 50 ps runs in which backbone restraints were gradually released from 10 to $5 \mathrm{kcal} / \mathrm{mol} \bullet \AA$ and ligands restraints released from 5 to $0.5 \mathrm{kcal} / \mathrm{mol} \bullet \AA$. These restraints were also kept for the following 100 ps equilibration in NVT ensemble and 100 ps in NPT 75 ensemble, then were gradually reduced to 0 during the following 400 ps of NPT equilibration. A 2 ns production run was then conduced on the unrestrained systems. A cut-off for electrostatic of $8 \AA$, a time step of 0.002 ps and the SHAKE algorithm, constraining bonds involving hydrogens, were applied to all ${ }_{80}$ calculation. ${ }^{27}$ The same protocol, extended to $4 \mathrm{~ns}$ of production run, was applied for the MD simulation of the $1 \beta, 1 \alpha-2 \beta, 2 \alpha$ tubulin tetramer (unbounded or bounded with 1-4), obtained by superposing two units of the previously described dimers to the tubulin tetramer crystal structure $3 \mathrm{~N} 2 \mathrm{G},{ }^{20}$ in order to preserve the 85 taxol binding site. Geometrical analyses and MM-PBSA calculations (50 snapshots at regular intervals) were performed in all cases on the last ns of the production run. Both Generalized Born and Poisson Boltzmann methods were applied for evaluating the electrostatic contribution to solvation energy, 90 obtaining comparable results (Table S1, Supporting Information). MD trajectory analyses were performed with both ptraj and VMD 1.8.5. ${ }^{28}$

Tubulin assembly assay. Tubulin was purified from bovine brain purchased from a local slaughterhouse, conserved before 95 use in ice-cold PBS (20 mM Na-phosphate, $150 \mathrm{mM} \mathrm{NaCl}, \mathrm{pH}$ 7.2) and used as soon as possible. Pure tubulin was obtained by two cycles of polymerization-depolymerization in a high-molarity buffer, ${ }^{29}$ resuspended in BRB80 (80 mM K-Pipes pH 6.9, 2 mM EGTA, $1 \mathrm{mM} \mathrm{MgCl}_{2}$ ), snap-frozen in aliquots in liquid nitrogen 100 and kept at $-80^{\circ} \mathrm{C}$. Protein concentration was determined by MicroBCA assay kit (Pierce). Stock solutions of the drugs were prepared by dissolving the powers at a concentration of $5 \mathrm{mM}$ in methanol. To assess their effects on tubulin assembly, bovine tubulin $(1.3 \mathrm{mg} / \mathrm{ml})$ was mixed with different compounds or an 105 equal volume of the solvent (final $1 \%$ methanol) in an assembly buffer minus GTP (80 mM K-Pipes pH 6.9, 2 mM EGTA, 1 mM $\mathrm{MgCl}_{2}, 10 \%$ glycerol). The reaction mixtures $(55 \mu \mathrm{l})$ were prepared at $0{ }^{\circ} \mathrm{C}$, and each reaction was started by plating the cuvette at $37{ }^{\circ} \mathrm{C}$ in an Ultraspec 300 spectrophotometer 110 (Pharmacia) equipped with a temperature controller. The kinetics of tubulin polymerisation were assessed by turbidimetry analysis following the increase in absorbance at $350 \mathrm{~nm}$ for $30 \mathrm{~min}$. At least three independent experiments were performed with each compound.

115 Kinetic parameters of tubulin assembly. The reaction of 
tubulin assembly has been analyzed to point out the potential effects of the selected compounds on the kinetic parameters defining its different phases: nucleation, elongation and steady state. Nucleation is a lag phase, during which a stable nucleus is 5 forming, and allows microtubule polymerization. From nucleation phase we extrapolated the parameter $p$ that represents the number of successive steps in the nucleation process by plotting $\log \left(\mathrm{A}(\mathrm{t}) / \mathrm{A}_{\infty}\right)$ against $\log t .^{30}$ Elongation develops after the lag phase and is the phase during which the maximum extent 10 of tubulin polymerization occurs following a procedure that is strongly similar to a first order chemical reaction. ${ }^{31}$ At very initial elongation phase, one can extrapolate the maximal velocity of polymerization $\left(\mathrm{V}_{\mathrm{i}}\right)$, as the variation of mass during time $(\mathrm{dA} / \mathrm{dt})$. Finally, steady state is the terminal phase during which the total 15 amount of microtubules does not change because the rates of microtubule growth and shortening are identical. We extrapolate the steady state extent of assembly $(\Delta \mathrm{A})$ as the absorbance maximum obtained at the plateau of the kinetics curve minus the absorbance measured at the initial elongation phase.

20 Differences between the effects of the different compounds on $p$ value, $\mathrm{V}_{\mathrm{i}}$ and $\triangle \mathrm{A}$ were evaluated by ANOVA followed by Dunnett post hoc test.

DIC microscopy. At the end of the assembly assay, microtubules were collected by centrifugation at $30,000 \mathrm{~g}$ for $30 \mathrm{~min}$ at $30{ }^{\circ} \mathrm{C}$, 25 fixed with $0.5 \%$ glutaraldehyde in the assembly buffer $(80 \mathrm{mM}$ K-Pipes pH 6.9, 2 mM EGTA, $1 \mathrm{mM} \mathrm{MgCl} 2,10 \%$ glycerol), and put onto coverslips. Image acquisition was performed using a Zeiss Axiovert 200 equipped with differential interference contrast (DIC) optics, a $63 \times$ oil objective, and a digital image 30 recording system (Axiocam HRM Rev. 2 camera driven by Axiovision software rel. 4.4, Zeiss).

Cell culture and immunofluorescence analyses. Microtubule organization in cell was revealed by indirect immunofluorescence (IF) analyses. Human breast adenocarcinoma cell line MCF7 35 (HTB-22; American Type Culture Collection, Rockville, MD, U.S.A.) was grown in minimal essential medium with Earle's (EMEM), supplemented with $10 \%$ fetal bovine serum (Hyclone Europe, Oud-Beijerland, Holland), $2 \mathrm{mM}$ L-glutamine, $100 \mathrm{U} / \mathrm{ml}$ penicillin and non-essential amino acids. Cells were maintained 40 at $37{ }^{\circ} \mathrm{C}$ in a humidified atmosphere at $5 \% \mathrm{CO}_{2}$. Experiments were carried out with cells plated on glass coverslips at a density of $1.0 \times 10^{4}$ cells $/ \mathrm{cm}^{2}$ and grown for $24 \mathrm{~h}$ in control medium following an incubation of $1 \mathrm{~h}$ and $72 \mathrm{~h}$ in the presence of the drugs (final concentration corresponding to the $\mathrm{IC}_{50}$ previously 45 determined by proliferation assay) ${ }^{18,19}$ or solvent vehicle alone (methanol) diluited 1:1000 in the culture medium. At the end of the treatments, cells were fixed and stained as previously described. ${ }^{32}$ Briefly, MCF7 cells were fixed and permeabilized for 6 min with methanol at $-20{ }^{\circ} \mathrm{C}$, washed with PBS and blocked 50 in PBS $+5 \%$ bovine serum albumin (BSA) for $15 \mathrm{~min}$ at room temperature. To localize tubulin, the cells were incubated with monoclonal anti $\alpha$-tubulin antibody (clone B-5-1-2, SigmaAldrich), 1:500 in PBS for $1 \mathrm{~h}$ at $37^{\circ} \mathrm{C}$. As secondary antibodies we used goat anti-mouse Alexa Fluor ${ }^{\mathrm{TM}} 568$ (Molecular Probes), ${ }_{55} 1: 1000$ in PBS $+1 \%$ BSA for $45 \mathrm{~min}$ at $37{ }^{\circ} \mathrm{C}$. Nuclei staining was performed by incubation with DAPI $(0.25 \mu \mathrm{g} / \mathrm{ml}$ in PBS $)$ for $15 \mathrm{~min}$ at room temperature. The coverslips were mounted in Mowiol ${ }^{\circledR}($ Calbiochem)-DABCO (Sigma-Aldrich) and examined with a Zeiss Axiovert 200 microscope equipped with a $63 \times$ ${ }_{60}$ Neofluor lens. Images were acquired with an Axiocam camera (Zeiss) and PC running Axiovision software (Zeiss).

\section{Acknowledgements}

We thank the "Consorzio Interuniversitario Lombardo per L'Elaborazione Automatica" (CILEA) for computational ${ }_{65}$ facilities. D.C. was supported by "Dote ricerca", FSE, Regione Lombardia.

\section{Notes and references}

${ }^{a}$ Dipartimento di Scienze Farmaceutiche - sezione di Chimica Generale e Organica "Alessandro Marchesini", Università degli Studi di Milano, via 70 Venezian, 2120133 Milano, Italy. Fax: +3902 50314476; Tel: +3902

50314480; E-mail: alessandro.contini@unimi.it

${ }^{b}$ Dipartimento di Bioscienze, Università degli Studi di Milano, via Celoria, 2620133 Milano, Italy

${ }^{c}$ Indena SPA, viale Ortles 12, 20139 Milano, Italy

$75+$ These authors equally contributed to this work.

† Electronic Supplementary Information (ESI) available: further computational and experimental details, extended tables, energies, Cartesian coordinates of the most stable geometries obtained for the unbounded ligands. See DOI: 10.1039/b000000x/

801 M. C. Wani, H. I. Taylor, M. E. Wall, P. Coggon, A. T. McPhail, J. Am. Chem. Soc., 1971, 93, 2325.

2 F. Gueritte-Voegelein, D. Guenard, F. Lavelle, M. -T. Le Goff, I. Mangatal, P. Potier, J. Med. Chem., 1991, 34, 992.

3 D. G. I. Kingston, J. Nat. Prod., 2000, 63, 726 and references cited 85 therein.

4 I. Ojima, J. C. Slater, S. D. Kuduk, C. S. Takeuchi, R. H. Gimi, C. M. Sun, Y. H. Park, P. Pera, J. M.Veith, R. J. Bernacki, J. Med. Chem., 1997, 40, 267.

5 M. Beer, L. Lenaz, D. Amadori, Ortataxel Study Group, J. Clin. Oncol., 2008, 26, May 20 suppl, abstr 1066.

6 a) A. Battaglia, E. Baldelli, E. Bombardelli, G. Carenzi, G. Fontana, M. L. Gelmi, A. Guerrini, D. Pocar, Tetrahedron, 2005, 61, 7727. b). E. Baldelli, A. Battaglia, E. Bombardelli, G. Carenzi, G. Fontana, M. L. Gelmi, A. Guerrini, D. Pocar, J. Org. Chem., 2004, 69, 6610. c) E. 95 Baldelli, A. Battaglia, E. Bombardelli, G. Carenzi, G. Fontana, A. Gambini, M. L. Gelmi, A. Guerrini, D. Pocar, J. Org. Chem., 2003, 68, 9773

7 a) J. Dubois, F. Guerritte-Voeglein, N. Guedira, P. Potier, B. Gilet, J. -C. Betoeil, Tetrahedron, 1993, 49, 6533. b) H. J. Williams, A. I. 100 Scott, R. A. Dieden, C. S. Swindell, L. E. Chirlian, M. M. Francl, J. M. Heerding, N. E. Krauss, Can. J. Chem., 1994, 72, 252. c) R. E. Cachau, R. Gussio, J. A. Beutler, G. N. Chmurny, B. D. Hilton, G. M. Muschik, J. W. Erickson, Int. J. Supercomput. Appl. High Perform. Comput., 1994, 8, 24.

1058 a) D. G. Vander Velde, G. I. Georg, G. L. Grunewald, C. W. Gunn, L. A. Mitscher, J. Am. Chem. Soc., 1993, 115, 11650. b) L. G. Paloma, R. K. Guy, W. Wrasidlo, K. C. Nicolau, Chem. Biol., 1994, 1, 107. c) I. Ojima, S. D. Kuduk, S. Chakravarty, M. Ourevitch, J. -P. Begue, J. Am. Chem. Soc., 1997, 119, 5519. d) I. Ojima, S. 110 Chakravarty, S. Lin, L. He, S. B. Horwitz, S. D. Kuduk, S. J. Danishefsky, Proc. Natl. Acad. Sci. U.S.A., 1999, 96, 4256.

9 a) L. Barboni, C. Lambertucci, G. Appendino, D. G. Vander Velde, R. H. Himes, E. Bombardelli, M. Wang, J. P. Snyder, J. Med. Chem. 2001, 44, 1576. b) T. C. Boge, Z. -J. Wu, R. H. Himes, D. G. Vander Velde, G. I. Georg, Bioorg. Med. Chem. Lett., 1999, 9, 3047. c) I. Ojima, S. Lin, T. Inoue, M. L. Miller, C. P. Borella, X. Geng, J. J. Walsh, J. Am. Chem. Soc., 2000, 122, 5343. d) I. Ojima, X. Geng, S. Lin, P. Pera, R. J. Bernacki, Bioorg. Med. Chem. Lett., 2002, 12, 349 352. e) X. Geng, M. L. Miller, S. Lin, I. Ojima, Org. Lett., 2003, 5 3733. f) O. Querolle, J. Dubois, S. Thoret, C. Dupont, F. Guéritte, D. Guénard, Eur. J. Org. Chem., 2003, 542. g) O. Querolle, J. Dubois, S. Thoret, F. Roussi, S. Montiel-Smith, F. Guéritte, D. Guénard, J. Med. Chem., 2003, 46, 3623, h) O. Querolle, J. Dubois, S. Thoret, F. 
Roussi, F. Guéritte, D. Guénard, J. Med. Chem., 2004, 47, 5937. i) R. Geney, L. Sun, P. Pera, R. J. Bernacki, S. Xia, S. B. Horwitz, C. L. Simmerling, I. Ojima, Chem. Biol., 2005, 12, 339.

10 a) T. Ganesh, R. C. Guza, S. Bane, R. Ravindra, N. Shaker, A. S.

5 Lakdawala, J. P. Snyder, D. G. I. Kingston, Proc. Nat. Ac. Sci., 2004, 101, 10006. c) S. Tang, C. Yang, P. Brodie, S. Bane, R. Ravindra, S. Sharma, Y. Jiang, J. P. Snyder, D. G. I. Kingston, Org. Lett., 2006, 8, 3983. d) T. Ganesh, C. Yang, A. Norris, T. Glass, S. Bane, R. Ravindra, A. Banerjee, B. Metaferia, S. L. Thomas, P. Giannakakou,

10 A. A. Alcaraz, A. S. Lakdawala, J. P. Snyder, D. G. I. Kingston, J. Med. Chem., 2007, 50, 713

11 a) J. P. Snyder, J. H. Nettles, B. Cornett, K. H. Downing, E. Nogales, Proc. Nat. Ac. Sci., 2001, 98, 5312-5316. b) A. A. Alcaraz, A. K. Mehta, S. A. Johnson, J. P. Snyder, J. Med. Chem., 2006, 49, 2478. c) Y. Paik, C. Yang, B. Metaferia, S. Tang, S. Bane, R. Rudravajhala, N. Shanker, A. A. Alcaraz, S. A. Johnson, J. Schaefer, R. D. O'Connor, L. Cegelski, J. P. Snyder, D. G. I. Kingston, J. Am. Chem. Soc., 2007, 129, 361.

12 M. Magnani, F. Ortuso, S. Soro, S. Alcaro, A. Tramontano, M. Botta, FEBS J., 2006, 273, 3301.

13 A. Mitra, D. Sept, Biophys. J., 2008, 95, 3252.

14 S. Pieraccini, G. Saladino, G. Cappelletti, D. Cartelli, P. Francescato, G. Speranza, P. Manitto, M. Sironi, Nature Chemistry, 2009, 1, 642.

15 J. Löwe,; H. Li, K. H. Downing, E. Nogales, J. Mol. Biol., 2001, 313, $25 \quad 1045-1057$

16 MOE 2010.10, http://www.chemcomp.com/

17 a) J. Wang, P. Morin, W. Wang, P. A. Kollman, J. Am. Chem. Soc., 2001, 123, 5221. b) N. Ferri, A. Corsini, P. Bottino, F. Clerici, A. Contini, J. Med. Chem., 2009, 52, 4087.

3018 I. Ojima, Y. H. Park, C. Sun, I. Fenoglio, G. Appendino, P. Pera, R. J. Bernacki, J. Med. Chem., 1994, 37, 1408.
19 a) G. Fontana, A. Battaglia, M. L. Gelmi, E. Baldelli, G. Carenzi, E. Bombardelli, C. Manzotti, R. J. Bernacki, SAR of 14-Substituted Taxanes. 228th ACS National Meeting, Philadelphia, PA, August 2235 26, 2004 Abstract MEDI \#99. b) See Supporting Information for experimental details.

20 P. Barbier, A. Dorleans, F. Devred, L. Sanz, D. Allegro, C. Alfonso, M. Knossow, V. Peyrot, J. M. Andreu, J. Biol. Chem., 2010, 285 , 31672.

4021 J. C. Gordon, J. B. Myers, T. Folta, V. Shoja, L. S. Heath, A. Onufriev, Nucleic Acids Res., 2005, 33, W368.

22 F. - Y. Dupradeau, A. Pigache, T. Zaffran, C. Savineau, R. Lelong, N. Grivel, D. Lelong, W. Rosanski, P. Cieplak, Phys. Chem. Chem. Phys., 2010, 12, 7821.

4523 Gaussian 03, Revision B.04, M. J. Frisch et al. Gaussian, Inc., Wallingford CT, 2004.

24 R. Bryce, http://www.pharmacy.manchester.ac.uk/bryce/amber/

25 D. A. Case, T. Cheatham, T. Darden, H. Gohlke, R. Luo, K. M. Merz, A. Onufriev, C. Simmerling, B. Wang, R. Woods, J. Comput. Chem., 2005, 26, 1668 .

26 J. Wang, R. M. Wolf, J. W. Caldwell, P. A. Kollman, D. A. Case, J. Comput. Chem., 2004, 25, 1157.

27 J. -P. Ryckaert, G. Ciccotti, H. J. C. Berendsen, J. Comput. Phys., 1977, 23, 327.

5528 W. Humphrey, A. Dalke, K. Schulten, J. Mol. Graph., 1996, 14, 33.

29 M. Castoldi, A. V. Popov, Proein Expr. Purif., 2003, 32, 83-88.

30 H. Flyvbjerg, E. Jobs, S. Leibler, Proc. Natl. Acad. Sci. USA, 1996, 93, 5975-5979.

31 K. A. Johnson, G. G. Borisy, J. Mol. Biol., 1977, 117, 1.

6032 G. Cappelletti, M. G. Maggioni, R. Maci, J. Neurosci. Res., 1999, 56, 28. 